University of New Mexico

UNM Digital Repository

Mathematics and Statistics Faculty and Staff

Publications

Academic Department Resources

2019

\title{
A short remark on Gödel incompleteness theorem and its self- referential paradox from Neutrosophic Logic perspective
}

\author{
Florentin Smarandache \\ University of New Mexico, smarand@unm.edu \\ Victor Christianto \\ victorchristianto@gmail.com
}

Follow this and additional works at: https://digitalrepository.unm.edu/math_fsp

Part of the Logic and Foundations Commons, Number Theory Commons, Other Mathematics Commons, and the Physics Commons

\section{Recommended Citation}

Smarandache, Florentin and Victor Christianto. "A short remark on Gödel incompleteness theorem and its self-referential paradox from Neutrosophic Logic perspective." International Journal of Neutrosophic Science (IJNS) (2019). https://digitalrepository.unm.edu/math_fsp/320

This Article is brought to you for free and open access by the Academic Department Resources at UNM Digital Repository. It has been accepted for inclusion in Mathematics and Statistics Faculty and Staff Publications by an authorized administrator of UNM Digital Repository. For more information, please contact amywinter@unm.edu, Isloane@salud.unm.edu, sarahrk@unm.edu. 


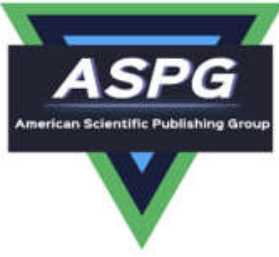

\title{
A short remark on Gödel incompleteness theorem and its self-referential paradox from Neutrosophic Logic perspective
}

\author{
V. Christianto ${ }^{1 *} \&$ F. Smarandache ${ }^{2}$ \\ ${ }^{1}$ Satyabhakti Advanced School of Theology - Jakarta Chapter, INDONESIA, \\ email: victorchristianto@gmail.com \\ 2 Dept. Mathematics \& Sciences, University of New Mexico, Gallup, USA. \\ Email: smarand@unm.edu \\ *Corresponding author: victorchristianto@gmail.com
}

\begin{abstract}
It is known from history of mathematics, that Gödel submitted his two incompleteness theorems, which can be considered as one of hallmarks of modern mathematics in $20^{\text {th }}$ century. Here we argue that Gödel incompleteness theorem and its self-referential paradox have not only put Hilbert's axiomatic program into question, but he also opened up the problem deep inside the then popular Aristotelian Logic. Although there were some attempts to go beyond Aristotelian binary logic, including by Lukasiewicz's three-valued logic, here we argue that the problem of self-referential paradox can be seen as reconcilable and solvable from Neutrosophic Logic perspective. Motivation of this paper: These authors are motivated to re-describe the self-referential paradox inherent in Godel incompleteness theorem. Contribution: This paper will show how Neutrosophic Logic offers a unique perspective and solution to Godel incompleteness theorem.
\end{abstract}

Keywords: Gödel incompleteness theorem, unprovability, undecidability, Neutrosophic Logic, Aristotelian Logic

\section{Introduction}

"This statement is unprovable." You can try to prove or disprove that particular statement, but indeed the statement is unprovable. That is how Gödel's incompleteness theorem began, see also [1], as in neutrosophic triplet: proved, disproved, unprovable (indeterminate). Try also another statement: "This statement is undecidable." Sounds 
interesting? It is in the particular logic of our language, the problem of unprovability and undecidability belong to true problems of Hilbert's axiomatic program.

According to Padula, which can be rephrased as follows [6]:

“Bertrand Russell and A. N. Whitehead's Principia Mathematica (1910-1913), in the future assigned as PM, contained a proof that the entire of arithmetic can be created based on set hypothesis. With it they wanted to demonstrate that all arithmetic is established on rationale. Kurt Gödel's confirmation (1931) of the 'inadequacy' of formal frameworks, for example, PM is significant for some reasons. It is significant throughout the entire existence of arithmetic and for additional improvements in science, for example, the hypothesis of calculations and the hypothesis of formal frameworks which has prompted the advancement of PCs and scripting languages, and advances towards man-made consciousness; for the development of scientific evidence and confirmation hypothesis; and for the improvement of rationale as it is educated today. It is fascinating in light of the fact that to ace it a comprehension of language is as significant as information on science."

In literature, there are expository works on that theorem, which is dubbed as one of the hallmarks of $20^{\text {th }}$ century mathematics. Rebecca Goldstein [2], wrote which can be paraphrased as follows:

The verification that was to turn into the "well known Incompleteness Proof" had clearly been cultivated the prior year, when Gödel was 23, and it was to be submitted in 1932 as his Habilitationsschrift, the last stage in the drawn out procedure of turning into an Austrian or German Dozent. It is one of the most surprising bits of numerical thinking at any point created, shocking both in the straightforwardness of its fundamental system and in the unpredictability of its subtleties, the meticulous making an interpretation of metamathematics into science by method of what has come to be called Gödel numbering. It is a completely requested mixing of a few layers of "voices," both scientific and metamathematical, contrast converging into symphonious harmonies at no other time heard. Music seems to give an especially adept similitude, which is the reason Ernest Nagel and James R. Newman in their great explicatory work, Gödel's Proof, portrayed the evidence as an "amazing intellectual symphony."

It is known, that the Neutrosophic Logic [8] is the only logic that can deal with the paradoxes, since a paradox P is a proposition that is true (its truth degree $\mathrm{T}=1$ ) and false (its false degree $\mathrm{F}=1$ ) in the same time, and as a consequence the paradox is also completely indeterminate (its indeterminate degree $\mathrm{I}=1$ ). Therefore, the Neutrosophic truth-values of the paradox is $\mathrm{P}(1,1,1)$, where $1+1+1=3>1$.

This paper will discuss, albeit shortly, on how Neutrosophic Logic can offer resolution to Gödel incompleteness theorem and its self-referential paradox.

\section{Background: what is formal axiomatic program?}

According to Steinmetz [3], which can be rephrased as follows: 
"A formal system is, basically, a framework that has been expressly and totally characterized. At its most fundamental level a proper framework comprises of a plainly characterized language. The language is involved an assortment of images that speak to the most crude components of the language and are utilized to build the equations of the framework alongside a rundown of decides that characterize what comprises a grammatically all around shaped or semantically important recipe. In this way, the depiction of the conventional framework is distinctive relying upon whether the proper framework is built from a proofhypothetical or a model-hypothetical point of view....

A proverbial framework is a framework that takes at least one recipes to be the maxims of the framework, which may possibly be a boundless number of equations if an adage diagram is utilized. The aphorisms of the framework are an assortment of recipes that are declared to be all around evident and from which the various genuine equations or hypotheses of the framework are gathered. In a proof-hypothetical framework the hypotheses of the framework are deductively demonstrated from the aphorisms of the framework or from recently demonstrated hypotheses. In a model-hypothetical framework the maxims of the framework characterize the substantial connections that exist between the articles that comprise the model of the framework and consequently the hypotheses of the framework are demonstrated dependent on what is valid for the items inside the model."

Into such a formal axiomatic program of Hilbert in early 1900, then came the young mathematician Gödel (see also [2][3][5]). What he did was to put the entire Hilbert's axiomatic program into question.

\section{Discussion on self-referential paradox and a principle of included middle}

Now, it is also possible to ask: how does Godel's incompleteness theorem give us a hint into what many physicists try to find: The Ultimate Theory or often dubbed as "TOE"? Ben-Yaacov wrote in his abstract, which can be rephrased as follows :

"An extreme Universal hypothesis - a total hypothesis that accounts, by means of not many and basic first standards, for all the marvels previously watched and that will ever be watched - has been, and still is, the desire of most physicists and researchers. However, an essential rule that is encapsulated in the aftereffects of Gödel's deficiency hypotheses is that self-referencing prompts consistent conflict or disappointment, as in the liar oddity or Russell's conundrum. In physical speculations self-referencing essentially happens when it is understood that the eyewitness is likewise a member in the accomplished marvels - we, people, are a piece of the Universe while watching it. In this manner self-referencing, and thusly intelligent conflicts, are unavoidable, and any hypothesis claiming to be Universal will undoubtedly be inadequate.’[4]

He also puts forth argument : 
"Does Gödel's theorem apply to physics ? A common argument in favour of applying Gödel's theorem to physics, is, more or less, that "Gödel's theorem applies to arithmetics which is the basis of mathematics, physics uses mathematics, therefore Gödel's theorem applies to physics."

Although there are counterarguments of the above statement, many problems with the advanced theoretical physics in the last 30-40 years seem to suggest that such is true for Gödel's theorem. With overreliance on heavy abstraction and sophisticated higher-mathematics, it became so hard to get our feet back to grounded (observed) realities - as proponents of grounded approach would say [7].

Now, after admitting this problem, then what is the resolution?

At this point, the following section will cite on how Neutrosophic Logic provides solution to the excluded middle principle in Aristotelian logic. According to one of us (FS):

"FS extended the Law of Included Middle $[<\mathrm{A}>,<$ nonA $>$, and a third value $<\mathrm{T}>$ which resolves their contradiction at another level of reality] to the Law of Included Multiple-Middle $[<\mathrm{A}>,<$ antiA $>$, and $<$ neutA $>$, where $<$ neutA $>$ is split into a multitude of neutralities between $<\mathrm{A}>$ and $<$ antiA $>$, such as $<$ neut $_{1} \mathrm{~A}>,<$ neut ${ }_{2} \mathrm{~A}>$, etc.]. The $<$ neutA $>$ value (i.e. neutrality or indeterminacy related to $<\mathrm{A}>$ ) actually comprises the included middle value. Also, he extended the Principle of Dynamic Opposition [opposition between $<\mathrm{A}>$ and $<$ antiA $>$ ] to the Principle of Dynamic Neutrosophic Opposition [which means oppositions among $<\mathrm{A}>,<$ antiA $>$, and $<$ neutA $>{ }^{1}$

Therefore there are more possibilities, beyond just excluded middle principle.

To summarize this discussion:

Godel incompleteness theorem actually exposes the fundamental problem in Aristotelian logic.That is excluded middle principle. As we may know, there are certain cases where paradoxes and even self-referential paradoxes exist.

So, in NL theory, it is always possible to find intermediate or third way::

$$
\text { (Standpoint A) -- intermediate/paradox - (Standpoint B) }
$$

But in NL theory we see those paradoxes in a new way, without rejecting it outright.

For example:

"This statement is unprovable."

\footnotetext{
${ }^{1}$ See FS's bio: http://fs.unm.edu/FlorentinSmarandache.htm, also url: http://fs.unm.edu/LawIncludedMultiple-Middle.pdf
} 
Or

"How do you decide between undecidability and unprovable?"

These two statements make Aristotelian logic defunct, but not Neutrosophic logic.

The Neutrosophic Logic is the only logic that can deal with the paradoxes, since a paradox P is a proposition that is true (its truth degree $\mathrm{T}=1$ ) and false (its false degree $\mathrm{F}=1$ ) in the same time, and as a consequence the paradox is also completely indeterminate (its indeterminate degree $\mathrm{I}=1$ ). Therefore, the Neutrosophic truth-values of the paradox is $\mathrm{P}(1,1,1)$, where $1+1+1=3>1$. No other logics allow the sum of its components to go over 1. Self-Referential Paradoxes have the same neutrosophic representation: $\mathrm{T}=1, \mathrm{~F}=1$, and $\mathrm{I}=1$.

\section{Concluding remarks}

This paper argues that Gödel incompleteness theorem and its self-referential paradox have not only put Hilbert's axiomatic program into question, but he also opened up the problem deep inside the then popular Aristotelian Logic. Although there were some attempts to go beyond Aristotelian binary logic, including Lukasiewicz's three-valued logic, here it is argued that the problem of self-referential paradox can be seen as reconcilable and solvable from Neutrosophic Logic perspective.

Summarizing, in Neutrosophic Logic, the Neutrosophic truth-values of the paradox is $\mathrm{P}(1,1,1)$, where $1+1+1=3>$ 1. No other logics allow the sum of its components to go over 1. Self-Referential Paradoxes have the same neutrosophic representation: $\mathrm{T}=1, \mathrm{~F}=1$, and $\mathrm{I}=1$.

Hopefully this article will inspire further investigations.

\section{References}

[1] K. Gödel. UBER FORMAL UNENTSCHEIDBARE S“ATZE DER "PRINCIPIA MATHEMATICA" UND VERWANDTER SYSTEME I, aus: “Monatshefte fur Mathematik und Physik” 38 (1931), 173-198.

[2] Rebecca Goldstein. Incompleteness: The Proof and Paradox of Kurt Gödel. New York: Atlas Books, 2005, pp. 156.

[3] Jason W. Steinmetz. An Intuitively Complete Analysis of Gödel's Incompleteness. arXiv: 1512.03667

[4] Uri Ben-Ya'acov. Gödel's incompleteness theorem and Universal physical theories. 2019 J. Phys.: Conf. Ser. 1391 012067. doi:10.1088/1742-6596/1391/1/012067

[5] Peter Smith. An introduction to Gödel's theorems. Second edition. Cambridge: Cambridge University Press, 2013.

[6] Janice Padula. The logical heart of a classic proof revisited: A guide to Gödel's 'incompleteness' theorems. Australian Senior Mathematics Journal 25 (1) 2011. 
[7] V. Christianto \& F. Smarandache. Lost in Mathematics: The Perils of Post-Empirical Science \& Their Resolution. In press

[8] F. Smarandache. A Unifying Field in Logics: Neutrosophic Logic. Neutrosophy, Neutrosophic Set, Neutrosophic Probability", American Research Press, Rehoboth, NM, 1999. 